\title{
BURAU REPRESENTATION AND RANDOM WALKS ON STRING LINKS
}

\author{
Xiao-Song Lin, Feng Tian and Zhenghan Wang
}

Using a probabilistic interpretation of the Burau representation of the braid group offered by Vaughan Jones, we generalize the Burau representation to a representation of the semigroup of string links. This representation is determined by a linear system, and is dominated by finite type string link invariants. For positive string links, the representation matrix can be interpreted as the transition matrix of a Markov process. For positive non-separable links, we show that all states are persistent.

\section{Beginning of the story: Burau representation.}

The Burau matrices $\beta_{i}, 1 \leq i \leq n-1$, are $n \times n$ matrices given as follows: Let $t \neq 0,1$ be a complex number. If we think of $\beta_{i}$ as a linear transformation on $\mathbb{C}^{n}$, then

$$
\beta_{i}=\underbrace{(1) \oplus \cdots \oplus(1)}_{i-1 \text { copies }} \oplus\left(\begin{array}{cr}
1-t & t \\
1 & 0
\end{array}\right) \oplus \underbrace{(1) \oplus \cdots \oplus(1)}_{n-i-1 \text { copies }},
$$

and

$$
\beta_{i}^{-1}=\underbrace{(1) \oplus \cdots \oplus(1)}_{i-1 \text { copies }} \oplus\left(\begin{array}{cc}
0 & 1 \\
\bar{t} & 1-\bar{t}
\end{array}\right) \oplus \underbrace{(1) \oplus \cdots \oplus(1)}_{n-i-1 \text { copies }} .
$$

Here we use $\bar{t}$ to denote $t^{-1}$ for simplicity.

It is easy to check that

$$
\begin{aligned}
& \beta_{i} \beta_{i+1} \beta_{i}=\beta_{i+1} \beta_{i} \beta_{i+1} \\
& \beta_{i} \beta_{j}=\beta_{j} \beta_{i} \quad \text { for }|i-j| \geq 2 .
\end{aligned}
$$

Thus, sending the standard generators $\sigma_{i}$ of the braid group $B_{n}$ to $\beta_{i}$ defines the (non-reduced) Burau representation of $B_{n}$. Obviously, the Burau representation fixes the column vector $(1, \ldots, 1)^{T}$ so we may have a reduced Burau representation acting on the quotient of $\mathbb{C}^{n}$ by $(1, \ldots, 1)^{T}$. We will 
see later in $\S 5$ that this is related with the probabilistic interpretation of the Burau representation we are going to explore.

For a given braid, its image under the Burau representation will be called the Burau matrix of that braid.

There is an extensive literature on the Burau representation. We only mention an article of John Moody [7] where it was proved (settling a question of long time) that the Burau representation is not faithful. In [5], Vaughan Jones offered a probabilistic interpretation of the Burau representation. We quote from [5] (with a small correction):

"For positive braids there is also a mechanical interpretation of the Burau matrix: Lay the braid out flat and make it into a bowling alley with $n$ lanes, the lanes going over each other according to the braid. If a ball traveling along a lane has probability $1-t$ of falling off the top lane (and continuing in the lane below) at every crossing then the $(i, j)$ entry of the (non-reduced) Burau matrix is the probability that a ball bowled in the $i$ th lane will end up in the $j$ th."

Let us now consider string links. This notion was first introduced in [4]. We will generalize it a little bit here and still call the generalization string links.

Essentially, a string link is an oriented tangle diagram (or simply a tangle) in the strip $\mathbb{R} \times[0,1]$ with bottom ends $\{1 \times 0,2 \times 0, \ldots, n \times 0\}$ (call them sources) and top ends $\{1 \times 1,2 \times 1, \ldots, n \times 1\}$ (call them sinks). There are exactly $n$ strands, each of them giving an oriented path from a bottom source $i \times 0$ to a top sink $j \times 1$. See Figure 1 . Two such string links are thought to be the same if they differ by a finite sequence of Reidemeister moves. As usual, this defines the same equivalence relation as isotopy of string links relative to the end points. Naturally, the set $S_{n}$ of all string links with $n$ strands has a semigroup structure such that $B_{n} \subset S_{n}$ is a subgroup.

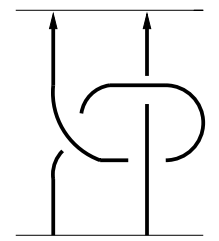

$T$

Figure 1. A favorable string link.

We now assign to each string link diagram with $n$ strands an $n \times n$ matrix whose entries will be shown to be rational functions in $t$ (see Theorem A in §2). Such an assignment is invariant under Reidermeister moves and 
multiplicative on $S_{n}$ so that we get a representation of the semigroup $S_{n}$ into the semigroup of $n \times n$ matrices (see Theorem B in $\S 2$ ).

Starting at the point $i \times 0$, we will try to walk up along strands of the given string link diagram $\sigma$ to get to the point $j \times 1$ according to the the following rules:

(1) The walking direction should always be in agreement with the orientation of strands of $\sigma$.

(2) If we come to a crossing on the lower segment, we will keep walking on the lower segment passing through that crossing.

(3) If we come to a crossing on the upper segment, we may choose either to jump down walking on the lower segment or keep walking on the upper segment passing through that crossing.

Such a way of walking from $i \times 0$ to $j \times 1$ on $\sigma$ is called a path.

A loop is a part of a path along which we may come back to where we start on a string link. A path is called simple if it contains no loops. Obviously, there are only finitely many simple paths on a string link. A loop is simple if it contains no other loops except itself. There are only finitely many simple loops on a string link. Any path can be reduced down to a simple path by dropping off simple loops it contains. Therefore, there are at most countably many paths on a string link.

Let us assign a weight $w(P)$ to a path $P$. Along $P$, there are many places where decisions are made about whether we jump down or keep walking. We will have a weight at each of these places along $P$ and $w(P)$ is the product of all weights on that path. The weights are determined as follows:

(1) If we come to a positive crossing on the upper segment, the weight is $1-t$ if we choose to jump down and $t$ otherwise; and

(2) If we come to a negative crossing on the upper segment, the weight is $1-\bar{t}$ if we choose to jump down and $\bar{t}$ otherwise.

With all these said, the $(i, j)$ entry of the $n \times n$ matrix assigned to the string link $\sigma \in S_{n}$ is

$$
\sum_{P} w(P)
$$

summing over all paths on $\sigma$ from the point $i \times 0$ to the point $j \times 1$. It is zero if there is no such a path. We will call the matrix $B(\sigma)$ with $(i, j)$-th entry defined above the Burau matrix of $\sigma$.

This paper extensively revises the first author's preprint "Burau representation and random walks on knots". He would like to thank the warm hospitality of the math department of the Chinese University of Hong Kong where this revision was worked out. 


\section{Basic properties of Burau matrices.}

In this section, we prove the following two basic theorems about the construction in $\S 1$.

Theorem A. The sum (1.1) converges to a rational function of $t$.

Theorem B. The matrix assigned to the string link diagram $\sigma \in S_{n}$ is invariant under Reidermeister moves. Moreover, this assignment determines a representation of the semigroup $S_{n}$ generalizing the Burau representation of the braid group $B_{n}$.

First we have the following observation.

We define the multiplicity of a path to be the number of simple loops it contains. There are only finitely many paths with a given multiplicity.

Observation. If $P$ is a path with multiplicity $k$, then

$$
w(P)=(1-t)^{k} \Phi(t)
$$

where $\Phi(t)$ is a formal power series of $1-t$. The sum (1.1) converges to a formal power series of $1-t$.

Proof. As formal power series of $1-t$, we have

$$
\begin{aligned}
& \bar{t}=1+(1-t)+(1-t)^{2}+\cdots+(1-t)^{n}+\cdots \\
& 1-\bar{t}=-(1-t)-(1-t)^{2}-\cdots-(1-t)^{n}-\cdots .
\end{aligned}
$$

Since a string link contains no closed components, the state of any simple loop contains a factor of $1-t$ or $1-\bar{t}$. This proves the first part.

Now the second part follows easily from the fact that there are only finitely many paths with a given multiplicity.

Let $F^{+}=F_{m}^{+}$be the free semigroup generated by $x_{1}, \ldots, x_{m}$. Let $\rho$ be a multiplicative function on $F^{+}$such that $\rho\left(x_{i}\right)$ is a rational function of $t$ for every $i=1, \ldots, m$. Moreover, assume that when $t=1, \rho\left(x_{i}\right)=0$.

Proposition 2.1. The sum

$$
\sum_{G \in F^{+}} \rho(G)
$$

converges to a rational function of $t$.

The convergence here means the existence of

$$
\lim _{n \rightarrow \infty} \sum_{G \in F^{+},|G| \leq n} \rho(G) .
$$


Proof. We prove inductively a stronger statement: The sum

$$
\Phi_{m}(t)=\sum_{G \in F_{m}^{+}} \rho(G)
$$

converges to a rational function of $t$ such that $\Phi_{m}(1)=1$. Actually,

$$
\Phi_{m}=\frac{1}{1-\sum_{i=1}^{m} \rho\left(x_{i}\right)} .
$$

This statement is certainly true when $m=1$ : Since $\rho\left(x_{1}\right)=0$ when $t=1$, for $|1-t|$ small enough, $\left|\rho\left(x_{1}\right)\right|<1$ and therefore

$$
\Phi_{1}(t)=\sum_{k=0}^{\infty} \rho\left(x_{1}^{k}\right)=\frac{1}{1-\rho\left(x_{1}\right)} .
$$

Obviously, $\Phi_{1}(t)$ is a rational function of $t$ such that $\Phi_{1}(1)=1$.

We list elements in $F_{m}^{+}$not equal to 1 as follows:

$$
\begin{aligned}
& G_{1} x_{m}^{k_{1}} G_{2} x_{m}^{k_{2}} \cdots G_{l} x_{m}^{k_{l}}, \\
& x_{m}^{k_{1}} G_{1} x_{m}^{k_{2}} G_{2} \cdots x_{m}^{k_{l}} G_{l}, \\
& x_{m}^{k_{0}} G_{1} x_{m}^{k_{1}} G_{2} x_{m}^{k_{2}} \cdots G_{l-1} x_{m}^{k_{l-1}}, \\
& G_{0} x_{m}^{k_{1}} G_{1} x_{m}^{k_{2}} G_{2} \cdots x_{m}^{k_{l-1}} G_{l-1},
\end{aligned}
$$

where $l=1,2, \ldots, k_{j}$ 's are positive integers and $G_{j}{ }^{\prime}$ are elements in $F_{m-1}^{+}$ not equal to 1.

Inductively, we have

$$
\begin{aligned}
& \sum_{k_{j}, G_{j}, l} \rho\left(G_{1} x_{m}^{k_{1}} G_{2} x_{m}^{k_{2}} \cdots G_{l} x_{m}^{k_{l}}\right) \\
& =\sum_{l=1}^{\infty}\left[\left(\Phi_{m-1}-1\right) \frac{\rho\left(x_{m}\right)}{1-\rho\left(x_{m}\right)}\right]^{l} .
\end{aligned}
$$

The last sum is convergent since $\Phi_{m-1}(1)-1=0$ and it is convergent to a rational function of $t$. Moreover, the sum is zero when $t=1$. The same conclusions hold for other kinds of terms in the above list of elements in $F_{m}^{+}$. Therefore, our inductive statement holds and this finishes the proof of Proposition 2.1. Moreover, a straightforward calculation will establish (2.1).

Proof of Theorem A. Multiple loops can be thought of as elements in the free semigroup generated by simple loops. We already observed that the multiplicative weight function $w$ on each simple loop is zero when $t=1$. 
Since there are only finitely many simple loops and finitely many simple paths, Theorem A follows immediately from Proposition 2.1.

Proof of Theorem B. For the first part, there are three types of Reidermeister moves. We check the invariance one by one. Notice that in Figure 2, 3 and 4 , the orientations of strands are determined by that of paths.

Type I: See Figure 2. For a positive kink, there are two paths (both simple) from the bottom end to the top end with $w=t$ and $w=1-t$ respectively. So total value is the same as no kink. For a negative kink, there is one simple path from the bottom end to the top end with $w=\bar{t}$. There is also a simple loop with $w=1-\bar{t}$. Therefore, the total value is

$$
\sum_{k=0}^{\infty} \bar{t}(1-\bar{t})^{k}=\frac{\bar{t}}{1-(1-\bar{t})}=1
$$

Again, this is the same as no kink.

Type II: See Figure 3. On the diagram on the left side of Figure 3, there are two paths going from the point $a$ to the point $b$ with $w=1-\bar{t}$ and $w=\bar{t}(1-t)$ respectively. Their sum is 0 , the same as no path from $a$ to $b$ on the diagram on the right side of Figure 3. Other situations can be checked similarly.

Type III: See Figure 4. On the diagram on the left side of Figure 4, there are two paths from $a$ to $b$ with $w=1-\bar{t}$ and $w=\bar{t}(1-t)^{2}=\bar{t}-2+t$ respectively. The total value is $t-1$, the same as the state $t(1-\bar{t})$ of the only path from $a$ to $b$ on the diagram on the right side. Other situations can be checked similarly.
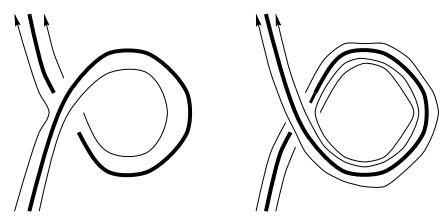

Figure 2. Type I Reidemeister move. 


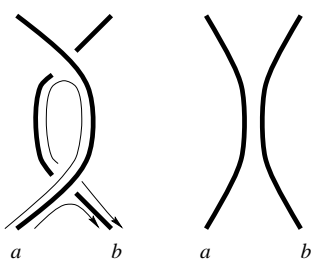

Figure 3. Type II Reidemeister move.
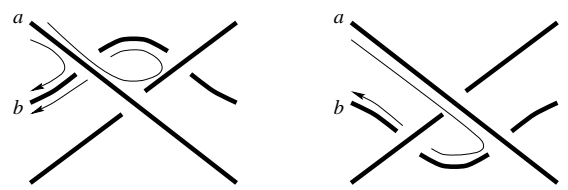

Figure 4. Type III Reidemeister move.

For the second part, simply notice that if we have two string links $\sigma_{1}$ and $\sigma_{2}$, a path on $\sigma_{1}$ from $i \times 0$ to $k \times 1$ followed by a path on $\sigma_{2}$ from $k \times 0$ to $j \times 1$ is a path on $\sigma_{1} \sigma_{2}$ from $i \times 0$ to $j \times 1$. Furthermore, the weight of paths is multiplicative. Therefore Burau matrices determine a representation of $S_{n}$. It is easy to see that this is a generalization of the Burau representation of $B_{n}$.

This finishes the proof of Theorem B.

Example. On the string link $T$ in Figure 1, there is only one simple loop with $w=t(1-\bar{t})$. There is one simple path for the entry $(1,1)$ with $w=1$, one simple path for the entry $(1,2)$ with $w=1-t$, and one simple path for the entry $(2,1)$ with $w=\bar{t}(1-t)$. All of them can be combined with multiple copies of the simple loop to form new paths. For the entry $(2,2)$, there are two simple paths with $w=t$ and $w=(1-t)^{2}(1-\bar{t})$ respectively. Only the second simple path can be combined with multiple copies of the simple loop to form new paths. The sum of the weights of all loops is convergent:

$$
\sum_{k=0}^{\infty}[t(1-\bar{t})]^{k}=\frac{1}{2-t}
$$

Therefore the Burau matrix of $T$ is

$$
\frac{1}{2-t}\left(\begin{array}{cc}
1 & 1-t \\
\bar{t}-1 & 3-t-\bar{t}
\end{array}\right)
$$




\section{Path structures of string links.}

In this section, we discuss the path structure of string links. This leads to an algorithm to compute our representation.

Given a string link $\sigma \in S_{n}$, we may encode all paths on $\sigma$ from a fixed bottom source to the top sinks into a binary tree. We first order all overcrossings on the string link from the first component to the last one following the orientations, and denote by $a_{k l}$ the $k$-th over-crossing on the $l$-th component. Then all paths from the bottom $i$-th source to the top sinks are in one-one correspondence with paths from $a_{1 i}$ in the following binary tree:

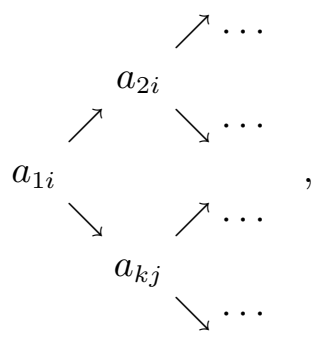

where the $a_{k i}$ following an uphill edge is the next over-crossing when we keep walking, and the $a_{k i}$ following a downhill edge is the next over-crossing when we jump down. The trees end when we arrive at a sink after an uphill or downhill edge. We have a total of $n$ trees, and each path (directed) in the tree is finite, but there are infinitely many paths.

Suppose $x, y$ are two points on the string link. Let $P(x, y)$ be the set of all paths from $x$ to $y$. Since all activities happen at over-crossings, $P(x, y)$ and $P\left(x^{\prime}, y^{\prime}\right)$ are naturally isomorphic if there are no over-crossings between $x, x^{\prime}$ and $y, y^{\prime}$. So in the following, we do not distinguish isomorphic path sets in this sense. In the case when a point on the string link is denoted by $a_{k l}$, it is the point on the $l$-th component right before the over-crossing $a_{k l}$.

Each path in $P(x, y)$ can be assigned a weight as before, and the total weight $w P(x, y)$ is the sum of the weights of all paths. Let us denote by $A_{k l}$ the row vector with $n$ components given by $\left(w P\left(a_{k l}, 1 \times 1\right), \ldots, w P\left(a_{k l}, n \times\right.\right.$ 1)). We have the following theorem.

Theorem C. The vectors $A_{k l}$ satisfy a linear system in the field of rational functions of $t$, and this linear system has a unique solution around $t=1$.

Proof. The linear system is given by the binary tree as follows: Before we arrive at the sinks, put $t^{\epsilon}$ on each uphill edge, and $1-t^{\epsilon}$ on each downhill edge, where $\epsilon= \pm 1$ is the sign of the corresponding over-crossing. At each vertex, say $a_{k l}$, followed by $a_{(k+1) l}$ (uphill), and $a_{m j}$ (downhill), the fundamental relation is

$$
A_{k l}=t^{\epsilon} A_{(k+1) l}+\left(1-t^{\epsilon}\right) A_{m j}
$$


In this relation, if the vertex $a_{k l}$ goes to a sink following an uphill edge, $A_{(k+1) l}$ should be replaced by the vector $(0, \ldots, 0,1,0, \ldots, 0)$ where 1 appears at the $l$-th position; and if $a_{k l}$ goes to a sink following a downhill edge, $A_{m j}$ should be replaced by the vector $(0, \ldots, 0,1,0, \ldots, 0)$ where 1 appears at the $j$-th position.

The existence of solutions follows from Theorem A. To prove the uniqueness, note that when we solve the linear system by eliminations, we need only to multiply equations by $t^{\epsilon}$ or $1-t^{\epsilon}$, and do substitutions.

As Burau matrix is given by $A_{1 i}, i=1, \cdots, n$ as rows, so the representation can be calculated by solving this linear system. For a string link with one component, all paths end at the same sink. So there are the obvious solutions $A_{k l}=1$. Hence, we have:

Corollary 1. If $\sigma \in S_{1}$, then $B(\sigma)=1$.

In general, we get a new linear system by summing all components in a vector. This leads to the following important corollary.

Corollary 2. If $\sigma \in S_{n}$, then $B(\sigma)$ always has 1 as an eigenvalue with an eigenvector $(1, \ldots, 1)^{T}$.

\section{Remarks.}

(1) If we exchange the role of sources and sinks, the representation matrix will change dramatically. In this sense, we say that our representation depends on the string link orientations.

(2) There are two obvious mirror images for string links: Imagine that a string link is laid almost flat in a horizontal plane. Then we have reflections in a vertical mirror or horizontal mirror, keeping the role of sources and sinks unchanged. In the vertical case, the matrix will change by $t \mapsto \bar{t}$. The other case is complicated.

(3) Theorems A and B can also be proved using Theorem C. Actually, $t^{\epsilon}$ and $1-t^{\epsilon}$ are the only possible coefficients in the fundamental relations which make the solution $A_{1 i}$ invariant under Reidermeister moves.

We can design an electrical device using a positive string link (all overcrossings are positive): We imagine that electrons enter from the bottom of a string link with a constant density, and at each over-crossing the current is branched to upper and lower segments with $t$ and $1-t$ percents of the entering density, respectively. Then the sum of each column in the Burau matrix gives the output current density at the top. If we reverse the process, so electrons enter from the top according to the output density function, we get a constant electric current. It seems to be an interesting question whether or not the output current functions are dense in an appropriate function space if we use all possible string links. 


\section{Dominance by finite type invariants.}

It we are allowed to have some crossings of a string link replaced by transverse double points, we get a singular string link. As usual, the Burau representation matrix can be extended canonically to singular string links. Given a string link $\sigma \in S_{n}$, and let $h=1-t$. Then we can expand $B(\sigma)$ into $\sum_{i=0}^{\infty} b_{k}(\sigma) h^{k}$. Obviously, $b_{k}$ are also invariants of string links. An invariant of string link is called of finite type of order $\leq k$ if it vanishes on any singular string link with more than $k$ double points. See $[\mathbf{1}, \mathbf{2}, \mathbf{6}]$.

Theorem D. The invariants $b_{k}$ is of finite type of order $\leq k$.

Proof. Let $\tau$ be a singular string link with $n>k$ double points and let $\sigma$ be the string link obtained from $\tau$ by resolving all double points to overcrossings. A path $P$ on $\sigma$ will induce paths on other resolutions of $\tau$, having zero weight if the induced path is illegal. The alternating sum of weights of all these induced paths is called the contribution of $P$ in $B(\tau)$. Thus, if a path on $\sigma$ does not pass through some double points on $\tau$, its contribution in $B(\tau)$ is zero. Otherwise, its contribution is zero modulo $h^{n}$. This could be seen easily as the weight difference of an over-crossing and an under-crossing is divisible by $h$.

This dominance of $B(\sigma)$ by finite type invariants is closely related with the following interesting fact: Although there might be infinitely many paths from a source to a sink, there are only finitely many with nonzero weights modulo $h^{n}$. In other words, if we restrict the number of jumping-downs, there are only finitely many paths from a source to a sink.

We don't know how to related our representation with the universal representation coming from iterated integrals [6].

\section{Markov processes on positive string links.}

Positive string links have only positive crossings. For such string links, the Burau representation could be used to determine Markov processes.

First let us recall some basic notions about Markov processes. All facts can be found in $[3]$.

Let $S$ be a finite or countable set. Suppose that to each pair $i, j$ in $S$ that is assigned a nonnegative number $p_{i j}$, and that these numbers satisfy the equations:

$$
\sum_{j \in S} p_{i j}=1, \quad i \in S
$$

Let $X_{0}, X_{1}, \ldots$ be a sequence of random variables which take values in $S$. The sequence is a Markov process if the conditional distribution of the next 
state $X_{n+1}$ given the present state $X_{n}$ is independent of the past states $X_{0}, \cdots, X_{n-1}$. The elements of $S$ are thought as the possible states of a system, $X_{n}$ representing the state at time $n$. The sequence or process $X_{0}, X_{1}, \ldots$ then represents the history of the system, which evolves in accordance with a probability law determined by the transition matrix $P=\left(p_{i j}\right)$, and the initial probabilities $\pi_{i}=P\left[X_{0}=i\right]$.

A matrix whose entries are nonnegative and satisfy Equation (5.1) is called a stochastic matrix. By an existence theorem (see [3], Theorem 8.1), given a stochastic matrix $P=p_{i j}$ and nonnegative numbers $\pi_{i}$ satisfying $\sum_{i} \pi_{i}=1$, there exists a Markov process on some probability space with initial probabilities $\pi_{i}$ and transition matrix $P$.

Given a positive string link, Corollary 2 in $\S 3$ tells us that for each $t \in$ $(0,1]$, the representation matrix is a stochastic matrix. Hence, given any initial conditions, there exists a Markov process with the components of the string link as the state space, and the transition matrix $B(\sigma)$. So we may imagine that a particle travels on a string link, and the state is the component it stays on. Each step it goes from one component (from bottom) to another (at top) with the probability equal to the total weight of all possible paths.

Let $p_{i j}^{(n)}$ be the $(i, j)$-th entry of the matrix $P^{n}$. A state $i \in S$ is persistent if

$$
\sum_{n} p_{i i}^{(n)}=+\infty
$$

Otherwise it is transient. All states are persistent if

$$
\sum_{n} p_{i j}^{(n)}=+\infty
$$

for all $i, j$. In this case, the system visits every state infinitely often.

A string link is separable if the link obtained by connecting the $i$-th source to the $i$-th sink (i.e., the usual closure of string links) can be separated by a 2 -sphere into two non-empty links.

Theorem E. Given a non-separable positive string link, then for each $t \in$ $(0,1)$ all states of the associated Markov process are persistent.

This means that walking randomly on a link which is the closure of a positive non-separating string link, we will visit every component of the link with probability 1.

We first have the following two lemmas. 
Lemma 1. If $\sigma \in S_{n}$ is a non-separable string link, then there exists an integer $N$ such that for every pair $i, j$, there is a path from the $i$-th source to the $j$-th sink in $\sigma^{N}$.

Lemma 2. Suppose $A$ is a stochastic matrix. If there exists an integer $N$ such that $a_{i j}^{(N)}>0$ for every pair $i, j$. Then

(1) 1 is the unique non-negative eigenvalue of $A$ with a strictly positive (row or column) eigenvector.

(2) No eigenvalue of $A$ has absolute value greater that 1.

(3) Let $u=\left(u_{1}, \ldots, u_{n}\right)$ be the unique row (left) eigenvector of $A$, then for each pair $i, j$,

$$
\lim _{n \rightarrow \infty} a_{i j}^{(n)}=u_{j}
$$

Lemma 2 follows from Perron-Frobenius theory and the renewal theorem (see pp. 16-17 of [8]). We first prove Theorem E, assuming Lemma 1 and Lemma 2.

Proof of Theorem E. By Lemma 1, for each pair $i, j$, we have a path from the $i$-th source to the $j$-th sink on $\sigma^{N}$. So for $t \in(0,1)$, the $(i, j)$-th entry in $B\left(\sigma^{N}\right)$ is positive. So, by Lemma 2,

$$
\lim _{n \rightarrow \infty} p_{i j}^{(n)}=u_{j}>0
$$

for each pair $i$ and $j$. It follows that

$$
\sum_{n} p_{i j}^{(n)}=+\infty
$$

This finishes the proof of Theorem E.

Proof of Lemma 1. We proceed by induction. The case $n=1$ is trivial.

Suppose our Lemma holds for $\leq n$ strands. For a string link $\sigma$ with $n+1$ strands, taking some power if necessary, we may assume that the permutation on $n+1$ letters induced by $\sigma$ is trivial so that the $i$-th strand connects the $i$-th source to the $i$-th sink. (Note: We don't claim that any power of a non-separable string link is still non-separable, although this seems to be true.) Delete the first strand and we get a string link with $n$ strands. The strands of this string link are partitioned into non-separable string links $\Delta_{k}$ with $\leq n$ strands. Since the induced permutation is the identity, powers of $\sigma$ will be partitioned into powers of $\Delta_{k}$. Now by taking some power of the string link $\sigma$ if necessary, we may assume inductively that each string link $\Delta_{k}$ has the property that any source and any sink 
is connected by a path. Since the string link $\sigma$ is non-separable, the first strand has to have over-crossings and under-crossings with strands in each $\Delta_{k}$. Then, it is easy to argue that on $\sigma^{N}$ for some $N$, any source and any sink can be connected by a path. This finishes the induction.

\section{Remarks.}

(1) As any link decomposes into non-separable links, it follows that for any positive string link, each state $i$ is persistent.

(2) When $t=1$, the stochastic matrix is a permutation matrix. When $t=0$, the limiting matrix is stochastic. But it seems to be hard to make a general statement.

(3) There is no difficulty to consider infinite string links. The situation is much like a 1-dimensional random walk. But they differs essentially. First our associated Markov process does not have spatial homogeneity. Secondly, the transition matrix is not local. It is possible for the particle to walk into any other position. It makes more sense to think of it as a "quantum 1-dimensional random walk".

(4) The existence of a row (left) strictly positive eigenvector corresponding to eigenvalue 1 makes the Markov process into a stationary distribution. So we can then define the entropy for the string link for each $t$ as in information

theory. It would be interesting to relate entropy with the complexity of the string link.

\section{References}

[1] D. Bar-Natan On the Vassiliev knot invariants, Topology, 34 (1995), 423-472.

[2] - Vassiliev homotopy string link invariants, Jour. of Knot Theory and its Ramifications, 4 (1995), 13-32.

[3] P. Billingsley, Probability and Measure, 2nd Edition, Wiley-Interscience, 1986.

[4] N. Habegger and X.-S. Lin, The classification of links up to link-homotopy, Jour. Amer. Math. Soc., 3 (1990), 389-419.

[5] V. Jones, Hecke algebra representations of braid groups and link polynomials, Ann. Math., 126 (1987), 335-388.

[6] X.-S. Lin, Power series expansions and invariants of links, in 'Geometric Topology' (Ed. W. Kazez), AMS/IP Studies in Adv. Math., Vol. 2, AMS \& International Press, 1996.

[7] J. Moody, The Burau representation of the braid group $B_{n}$ is unfaithful for large $n$, Bull. Amer. Math. Soc. (N.S.), 25 (1991), 379-384. 
[8] P. Walters, An Introduction to Ergodic Theory, GTM, Vol. 79, Springer-Verlag, 1982.

Received June 1, 1996 and revised January 15, 1997. The third author is supported by an NSF Postdoctoral Fellowship.

UNIVERSITY OF CALIFORNIA

RIVERSIDE, CA 92521-0135

E-mail address: xl@math.ucr.edu

University of California, San Diego

LA JOLLA, CA 92093

E-mail address: ftian@euclid.ucsd.edu

University of CALifornia, San Diego

LA Jolla, CA 92093

AND

INDIANA UNIVERSITY

BloOMington, IN 47405

E-mail address: zwang@euclid.ucsd.edu

Note: The reference at the Beginning of the proof of Theorem B to Figures 2, 3, AND 4 APPEARED IN THE PAPER VERSiON AS "Figures 3, 4, AND 5." 